\title{
Functional Subregions in Primary Auditory Cortex Defined by Thalamocortical Terminal Arbors: An Electrophysiological and Anterograde Labeling Study
}

\author{
David S. Velenovsky, ${ }^{1}$ Justin S. Cetas, ${ }^{1}$ Robin 0. Price, ${ }^{1}$ Donal G. Sinex, ${ }^{2}$ and Nathaniel T. McMullen ${ }^{1}$ \\ ${ }^{1}$ Department of Cell Biology and Anatomy, University of Arizona College of Medicine, Tucson, Arizona 85724, and ${ }^{2}$ Department of Speech and Hearing \\ Science, Arizona State University, Tempe, Arizona 85287
}

Several functional maps have been described in primary auditory cortex, including those related to frequency, tuning, latency, binaurality, and intensity. Many of these maps are arranged in a discontinuous or patchy manner. Similarly, thalamocortical projections arising from the ventral division of the medial geniculate body to the primary auditory cortex are also patchy. We used anterograde labeling and electrophysiological methods to examine the relationship between thalamocortical patches and auditory cortical maps. Biotinylated dextran-amine was deposited into physiologically characterized sites in the ventral division of the medial geniculate body of New Zealand white rabbits. Approximately $7 \mathrm{~d}$ later, the animal was again anesthetized and the ipsilateral auditory cortex was mapped with tungsten microelectrodes. Multi-unit physiological data were obtained for the following characteristics: best frequency (BF), binaurality, response type, latency, sharpness of tuning, and threshold. Immunocytochemical methods were used to reveal the injection site in the ventral division of the medial geniculate body as well as the anterogradely labeled thalamocortical afferents in the auditory cortex. In $86 \%$ of the cases (12 of 14), entry into a thalamocortical patch was associated with a marked change in physiological responses. A consistent BF and binaural class were usually observed within a patch. The patches appear to innervate distinct functional regions coding frequency and binaurality. A model is presented showing how patchy thalamocortical projections participate in the formation of tonotopic and binaural maps in primary auditory cortex.

Key words: medial geniculate body; audition; neocortex; frequency map; thalamus; patches

\section{Introduction}

The topographic representation of peripheral sensory receptors in mammalian sensory systems results in a systematic representation or functional map in sensory cortex (Marshall et al., 1941; Hubel and Wiesel, 1962; Merzenich et al., 1973; Hubel and Wiesel, 1977). The first tonotopic auditory neocortical map was demonstrated by Woolsey and Walzl (1942) in the form of a cochleotopic organization in the primary auditory cortex (AI) of the cat. Other functional maps in AI include binaural interaction bands (Imig and Adrian, 1977; Middlebrooks et al., 1980), threshold/intensity (Tunturi, 1950; Schreiner et al., 1992), and sharpness of tuning (Schreiner and Mendelson, 1990). Interestingly, these maps are patchy or discontinuous in their distribution relative to the frequency map (Merzenich et al., 1973; Brugge and Imig, 1978; Middlebrooks et al., 1980; Reale and Imig, 1980;

\footnotetext{
Received Aug. 27, 2002; revised 0ct. 16, 2002; accepted 0ct. 17, 2002.

This work was supported by National Institutes of Health (NIH)/National Institute of Neurological and Communicative Disorders and Stroke Grants DC05108 (D.S.V.) and DC02410 (N.T.M.) and a Deafness Research Foundation Award (D.S.V.). J.S.C. was the recipient of a Predoctoral Fellowship from NIH Training Grant NS07434. R.O.P. was supported by the Undergraduate Biology Research Program at the University of Arizona and the Howard Hughes Medical Institute. We thank Dr. Naomi Rance for useful comments on a previous version of this manuscript, Jeb Zirato for his help with the photomicroscopy, and Vanessa Lopez for assistance with generation of computer reconstructions of thalamocortical patches.

Correspondence should be addressed to Dr. D. S. Velenovsky, Department of Cell Biology and Anatomy, University of Arizona College of Medicine, P.0. Box 245044, 1501 North Campbell Avenue, Tucson, AZ 85724. E-mail: dsv@u.arizona.edu.

Copyright $\odot 2002$ Society for Neuroscience $\quad 0270-6474 / 02 / 220308-09 \$ 15.00 / 0$
}

Redies et al., 1989; Schreiner, 1991, 1992; Schreiner et al., 1992, 2000; Mendelson et al., 1993, 1997; Fitzpatrick et al., 1998).

Ascending auditory signals reach AI via projections from the ventral division of the medial geniculate body (MGV) (Mesulam and Pandya, 1973; Sousa-Pinto, 1973; Jones and Burton, 1976; Winer et al., 1977; Imig and Morel, 1983; Middlebrooks and Zook, 1983; LeDoux et al., 1985). Anterograde studies of MGV projections have revealed the existence of a discontinuous or patchy projection of afferent axons from the MGV to layers III/IV of auditory cortex (McMullen and de Venecia, 1993; Romanski and LeDoux, 1993; de Venecia and McMullen, 1994; Cetas et al., 1999). Patchy thalamocortical (TC) afferents to the cortex have been described in a variety of species, including the rat, rabbit, ferret, cat, and macaque (Winer et al., 1977; Angelucci et al., 1993; Romanski and LeDoux, 1993; Hashikawa et al., 1995; Huang and Winer, 2000). Some insight into the nature of TC patches has come from studies involving the calcium-binding protein parvalbumin (PV) (Hendry et al., 1989; Morino-Wannier et al., 1992; McMullen et al., 1994; de Venecia et al., 1995, 1998; Jones et al., 1995; Molinari et al., 1995). TC patches coexist with PV-positive patches in AI (de Venecia et al., 1998). In addition, the MGV is intensely PV-positive in many species (Hashikawa et al., 1991; Vater and Braun, 1994; de Venecia et al., 1995; Molinari et al., 1995; Cruikshank et al., 2001), and PV-positive relay neurons in the MGV project to AI (de Venecia et al., 1998). Thus, at least some of the TC patches arising from the MGV may represent a chemically coded pathway to AI. Evidence that specific TC circuits contribute to patchy functional maps in $\mathrm{AI}$ was presented by 
Middlebrooks and Zook (1983), who demonstrated that excitatory/inhibitory (EE/EI) foci in AI receive input from segregated populations of neurons in the auditory thalamus. Surprisingly, the physiological responses of neurons within TC patches and their relationship to various acoustic maps existing in AI have not been addressed in any species. In the present study, this question was examined by mapping the auditory cortex in animals that received injections of anterograde tracer into the ventral division of the medial geniculate body. Portions of this work have been published previously in abstract form (Velenovsky et al., 2000, 2001).

\section{Materials and Methods}

Animals. The animals were housed, cared for, and used strictly in accordance with United States Department of Agriculture regulations and the Guide for the Care and Use of Laboratory Animals (National Institutes of Health publication 85-23). The research protocol was approved by the Institutional Animal Care and Use Committee of the University of Arizona College of Medicine. Experiments were performed on normal young adult New Zealand white (NZW) rabbits (1-3 kg) obtained from local suppliers. The experiments were performed in two parts. In part I, an anterograde tracer was injected into the ventral division of the MGB and the animal was allowed to recover. In part II, the ipsilateral auditory cortex was electrophysiologically mapped with microelectrodes $6-8 \mathrm{~d}$ later. All labeling and mapping procedures were performed in an IAC model 401A sound booth (Industrial Acoustics Company, Bronx, NY).

Part I: anterograde labeling of TC pathway. Animals were anesthetized with ketamine $(44 \mathrm{mg} / \mathrm{kg}$, i.m.) and xylazine $(10 \mathrm{mg} / \mathrm{kg}$, i.m.) and then placed in a Kopf stereotaxic device (David Kopf Instruments, Tujunga, CA). Surgery was performed under sterile conditions. Injection sites were referenced to bregma (lambda and bregma in the same horizontal plane) using stereotaxic coordinates (McMullen and de Venecia, 1993). The final coordinate ranges were as follows: anteroposterior, $6.5-7.2 \mathrm{~mm}$; lateral, 5.3-5.6 mm; dorsoventral, 12.25-13.65 (referenced from skull surface). After small holes were drilled through the skull, tungsten electrodes (2-4 M $\Omega$; World Precision Instruments, Sarasota, FL) were advanced stereotaxically into the auditory thalamus using a Kopf micromanipulator fitted with a motorized stage (model MC-3B-11; National Aperture Inc., Salem, NH). Custom-fabricated transducers coupled to custom-molded earpieces were used to deliver acoustic stimuli. TuckerDavis Technologies (Gainesville, FL) system II hardware controlled by a Gateway (Poway, CA) personal computer using custom software were used to generate auditory stimuli and analyze multi-unit responses as the electrode was advanced in $100 \mu \mathrm{m}$ steps. Multi-unit responses were amplified and filtered using a Grass P511 preamplifier (Grass Instruments, West Warwick, RI). After noise bursts (one per second; 100-200 msec duration) revealed an acoustically responsive area, tone bursts (one per second; $100-200 \mathrm{msec}$ duration) were used to characterize best frequency $(\mathrm{BF})$, latency, and response profile of the site. When shortlatency-onset responses and sharp tuning curves in response to tone bursts were seen, the coordinates were noted and the electrode was slowly removed. A borosilicate glass electrode (outside tip diameter, $10-13 \mu \mathrm{m}$ ) filled with $10 \%$ biotinylated dextran-amine (BDA, molecular weight of 10,000; Molecular Probes, Eugene, OR) in $0.1 \mathrm{~m}$ PBS was returned to the MGB recording site. This was accomplished by placing the injection microelectrode at the bregma reference site under high magnification. The identical stereotaxic coordinates of the tungsten electrode were used to locate the injection electrode at the original recording site. The BDA was deposited using positive current pulses $(0.9 \mathrm{~Hz}, 3 \mu \mathrm{A}$ for $20 \mathrm{~min})$ with a Transkinetics CS-3 current generator. After the electrode was removed, the incision was sutured, and chloromycetin $(30 \mathrm{mg} / \mathrm{kg}$, s.c.) was administered. The animal was returned to its cage after it recovered from anesthesia and was monitored daily for signs of infection or discomfort.

Part II: mapping of TC patches. After 6-8 d, the animal was returned to the recording room and to the Kopf stereotaxic device for cortical mapping. The animal was anesthetized using urethane $(1.25 \mathrm{mg} / \mathrm{kg}$, i.v. $)$ and ketamine ( $13 \mathrm{mg} / \mathrm{kg}$, i.m., every $50 \mathrm{~min})$. Oval holes of $\sim 4 \times 6 \mathrm{~mm}$ were drilled through the skull just dorsal to AI. Tangential penetrations were made through the auditory cortex (McMullen and Glaser, 1982) with tungsten microelectrodes (2-4 M $\Omega$; World Precision Instruments). Multi-unit response properties were determined at $300 \mu \mathrm{m}$ intervals. This recording interval was large enough to maximize cortical mapping but remained fine enough to permit functional/anatomical correlations. Broadband noise bursts were used to determine the overall responsiveness of an area to auditory stimuli. When a responsive area was located, the characteristic frequency, threshold, and binaurality were determined using tone bursts (100 msec duration). Binaurality was determined by presenting noise bursts at a level $20-40 \mathrm{~dB}$ above threshold. For binaural presentation, diotic stimuli were used. Responsiveness was judged by listening to multi-unit activity through a monitor speaker and viewing real-time spike rasters. Responses were classified as EE if diotic stimuli elicited the most robust response and EI if stimulating the contralateral ear elicited a more robust response. No preference for contralateral or binaural stimulation was classified as excitatory/occlusion (EO) (Imig and Adrian, 1977; Middlebrooks and Zook, 1983). Afterward, spike rasters, poststimulus time histograms (PSTHs) and tuning curves were obtained (Cetas et al., 2002a). The binaurality determined for noise bursts was used to set the mode of stimulus presentation (contra or binaural) when the automated tuning curve program was run. The BF from the tuning curve program was then evaluated for binaurality, using levels and procedure as for noise above (Cetas et al., 2001). Electrolytic lesions (10 $\mu \mathrm{A}$ for $10 \mathrm{sec}$ ) were placed at the end of each penetration and at the location of the initial acoustic response to assist in electrode track reconstructions. At completion of the mapping experiments, the animal was perfused with $4 \%$ paraformaldehyde in PBS and the brain was removed. The brain was dissected into cortical and thalamic blocks and postfixed overnight in $4 \%$ paraformaldehyde at $4^{\circ} \mathrm{C}$. The blocks were cryoprotected in ascending sucrose solutions (to $30 \%$ ) before sectioning.

Tissue processing. For the localization of injection sites, coronal thalamic sections were cut using a sledge-type microtome (75 $\mu \mathrm{m}$ thick) and incubated for $15 \mathrm{~min}$ in $1 \% \mathrm{H}_{2} \mathrm{O}_{2}$ to suppress endogenous peroxidase activity. BDA was localized by avidin-biotin-horseradish peroxidase histochemistry (Vector Elite ABC Kit; Vector Laboratories, Burlingame, CA) using nickel-cobalt intensification of the diaminobenzidine (DAB) reaction product (Adams, 1981). Sections were mounted on gelatinized slides and counterstained with $1 \%$ aqueous methylene blue to confirm the location and extent of the MGV injections. The electrophysiologically mapped cortices were gently flattened between glass slides and fixed in $4 \%$ paraformaldehyde. Tangential frozen sections through the cortex were cut on a sledge-type microtome at a thickness of $50 \mu \mathrm{m}$. A sensitive immunoperoxidase method was used to visualize BDA-labeled axons in the cortex ipsilateral to MGV injections (McMullen and de Venecia, 1993; de Venecia and McMullen, 1994). Briefly, sections were incubated for $48 \mathrm{hr}$ at $4^{\circ} \mathrm{C}$ in goat anti-biotin antibody (Vector Laboratories) diluted 1:10,000 in PBS containing 3\% normal rabbit serum (NRS), followed by biotinylated rabbit anti-goat IgG (Vector Laboratories) diluted 1:200 in 3\% NRS-PBS for $2 \mathrm{hr}$ at room temperature. The labeled axons were visualized with a standard DAB histochemistry reaction with heavy metal intensification (Adams, 1981). Because PV has been shown to be an excellent marker for AI in a variety of species (Wallace et al., 1991; McMullen et al., 1994; Hashikawa et al., 1995; Kosaki et al., 1997; de Venecia et al., 1998; Budinger et al., 2000; Cruikshank et al., 2001), alternative cortical sections were processed for this calcium-binding protein. The monoclonal antibody against PV used in these experiments was Swiss Antibodies \#235 (Swant, Bellinzona, Switzerland). All immunohistochemical procedures were performed on free-floating sections using a rocker table for gentle agitation. Dilutions and rinses were done with 0.1 M PBS, pH 7.4. Sections were first submerged in $1 \% \mathrm{H}_{2} \mathrm{O}_{2}$ for 15 min to suppress endogenous peroxidase activity and then placed into $3 \%$ normal horse serum (NHS; Vector Laboratories) with 1\% Triton X-100 for $60 \mathrm{~min}$ to block nonspecific labeling and to increase antibody penetration. The sections were incubated in primary mouse monoclonal antibody to PV (1:5000-1:15,000 dilution with $3 \%$ NHS) for $72 \mathrm{hr}$ at $4^{\circ} \mathrm{C}$, followed by biotinylated horse anti-mouse $\operatorname{IgG}$ (1:200 dilution with $3 \%$ NHS) for $2 \mathrm{hr}$, and avidin-biotin-horseradish peroxidase complex 

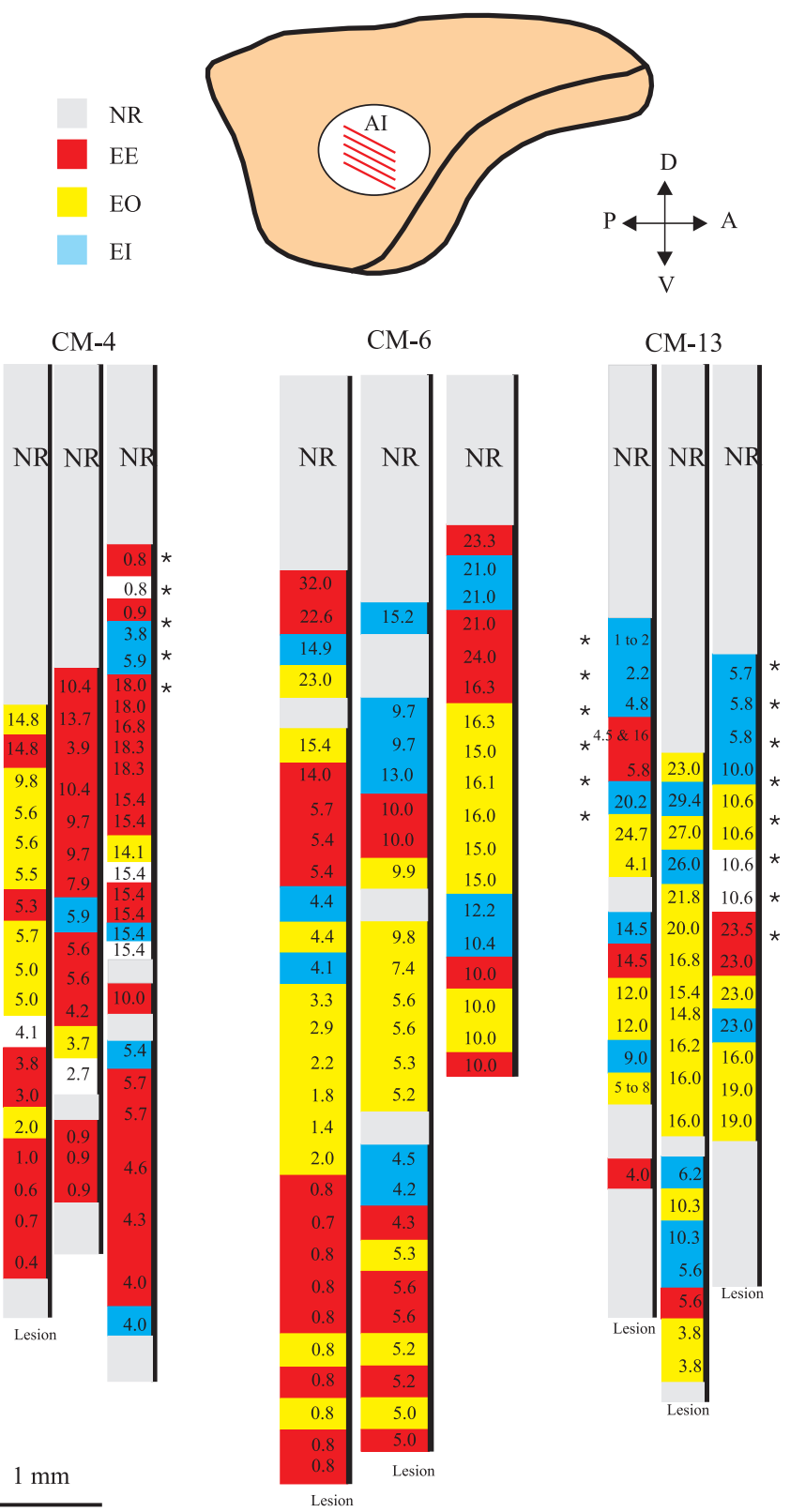

Figure 1. Reconstruction of frequency and binaural maps from three animals (CM-4, $C M-6$, and (M-13). Gray shaded areas represent regions of no response (NR) to acoustic stimuli. The numbers represent $\mathrm{BF}$ in kilohertz at each recording site. Binaural classes (EE, Red; $\mathrm{E} 0$, yellow; $\mathrm{El}$, blue) are also indicated. Note the clusters of similar binaural classes in each penetration. The reverse (low-high) tonotopic progression in the dorsal region of CM-4 and CM-13 (asterisks) represents a second auditory field. Inset, Side view of the rabbit brain showing the location of the $\mathrm{Al}$ and the orientation of isofrequency contours. $A$, Anterior; $D$, dorsal; $P$, posterior; $V$, ventral.

(Vector Laboratories Standard ABC kit) for $90 \mathrm{~min}$ at room temperature. PV immunoreactivity was visualized using the cobalt-nickel DAB intensification method of Adams (1981). No specific staining was observed in control experiments in which sections were incubated in primary antibody preadsorbed with HPLC-purified rat parvalbumin (Swiss antibodies). All sections were then mounted onto gelatinized slides and coverslipped with Permount. TC patches, electrode tracks, and electrolytic lesions were reconstructed from serial sections with the aid of a computer microscope (MicroBrightField Inc., Colchester, VT) or an Aus Jena Macro-Projector (AusJena, Jena, Germany). Tissue shrinkage was determined by measuring the distances between lesions after tissue processing and comparing those values with those measured in situ. A shrinkage measurement of $30 \%$ was determined and applied to reconstruct the cortical electrode penetrations.

\section{Results}

All successful injections into the MGV resulted in labeled TC patches. AI was mapped in 12 animals, with a total of 35 tangential electrode penetrations. In seven animals, at least one TC patch was traversed by a mapping penetration. In six of the seven animals, the BF of the MGV injection site was determined. In three of the six animals, BF and binaurality of the injection site were represented in at least one TC patch. Reconstructions of frequency and binaural maps obtained from tangential electrode penetrations in three animals are shown in Figure 1. In all cases in which AI was mapped, a high-to-low dorsoventral frequency progression was seen. In 16 penetrations, an additional tonotopic field was located dorsal and often anterior to the primary field, with a low-to-high frequency progression that mirrored the progression in the primary field. This secondary field is shown for animals CM-4 and CM-13 in Figure 1 (denoted by asterisks) and CM-15 in Figure 3. The distribution of binaural categories relative to the tonotopic maps is also shown in Figure 1. In general, neurons formed clusters of binaural categories that extended across isofrequency contours. EE and EO categories predominated and formed regions $0.5-2.0 \mathrm{~mm}$ wide. EI regions were seen less frequently.

A total of 14 BDA-labeled TC patches were mapped in seven animals. Typically, multiple TC arbors in AI resulted from a single BDA injection in the MGV (Fig. 2). In many cases, the TC patches in the tangential plane appeared oriented along the presumptive isofrequency contours in AI (shown in Fig. 1) of the rabbit (see Figs. 2, 3, 5, 7, 9). In all but one case, labeled patches arising from MGV tracer injections coincided with tonotopically organized areas in auditory cortex, whose functional characteristics (short latency, low threshold, dorsoventral high-to-low frequency organization) were consistent with AI of this species (McMullen and Glaser, 1982). In 86\% (12 of 14 in seven animals) of the penetrations through labeled TC patches, entry and exit into anterograde-labeled terminal fields were characterized by abrupt

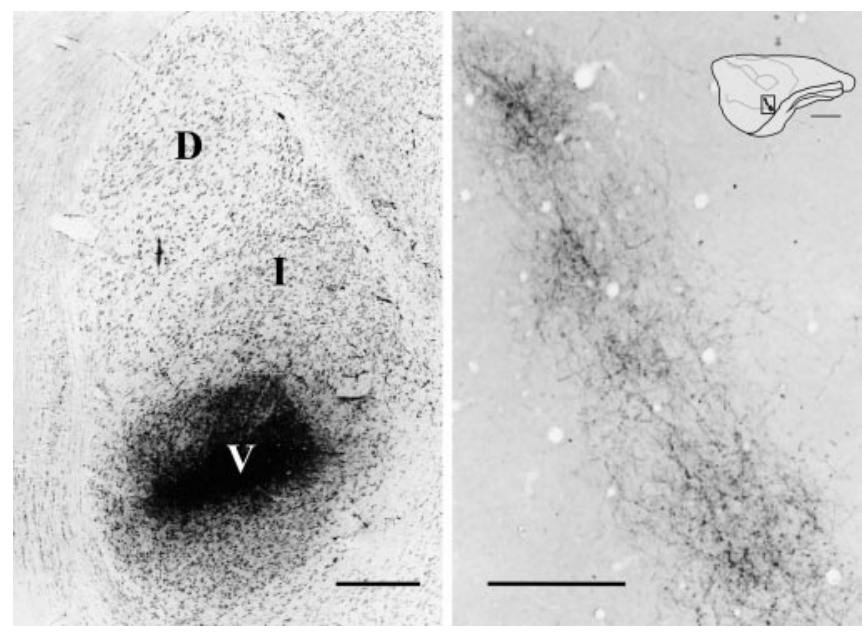

Figure 2. Thalamic BDA injection and anterograde-labeled TC fields. Left, Methylene blue counterstained coronal section through the right MGV showing large BDA injection ventral division $(V)$ of the MGV. The injection is elongated parallel to the MGV cellular laminas, which exhibit a dorsomedial to ventrolateral orientation. Dorsal $(D)$ and internal $(I)$ MGV subdivisions are also visible. Scale bar, $500 \mu \mathrm{m}$. Right, Tangential view of four TC patches labeled by the BDA injection shown to the left. Scale bar, $1.0 \mathrm{~mm}$. Inset, Side view of the rabbit brain. The boxed region shows the location of labeled TC patches in Al. Scale bar, $5.0 \mathrm{~mm}$. 


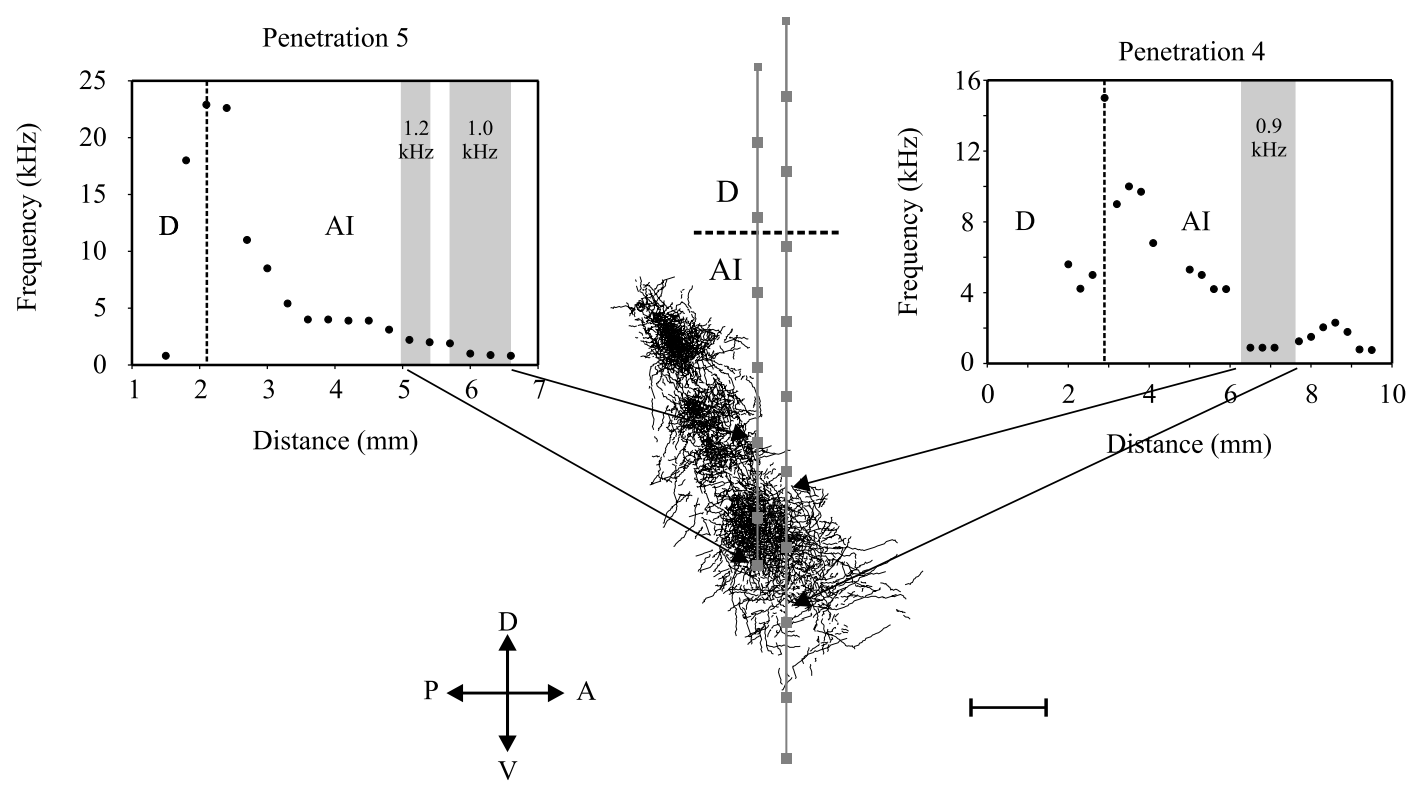

Figure 3. Reconstruction of two mapping penetrations superimposed on TCterminal field axons arising from the BDA injection shown in Figure 2. Graphs depict BF as a function of electrode depth throughout tangential penetrations 4 and 5. Dotted lines indicate the BF reversal at the border between the dorsal field $(D)$ and Al. The shaded area in each graph represents the portion of the penetration that passed through a TC terminal field. Note the consistent BF within the patches and the change in frequency as each electrode enters or exits the TC terminal fields. $A$, Anterior; $V$, ventral; $P$, posterior. Scale bar, $1.0 \mathrm{~mm}$.

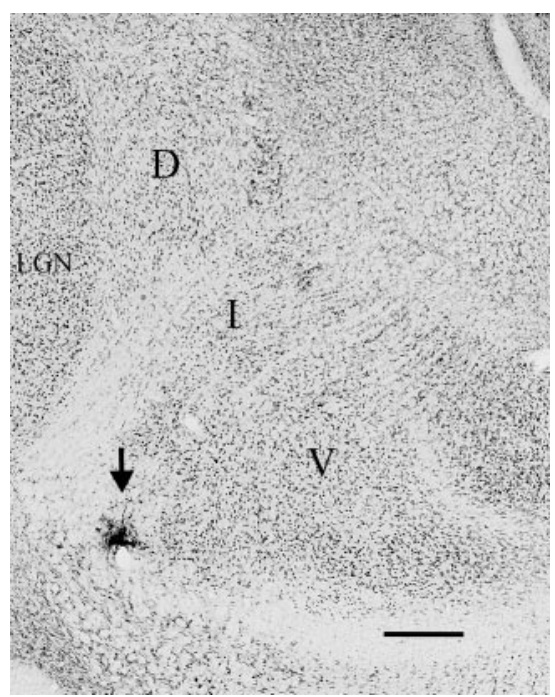

Figure 4. Methylene blue counterstained coronal section through the right MGB in experiment CM-27, showing a small BDA injection (arrow) in the far lateral MGV (V). D, Dorsal subdivision of the MGB; I, internal division of the MGB; $L G N$, lateral geniculate nucleus. Scale bar, $500 \mu \mathrm{m}$.

changes in physiological responses (e.g., binaurality, latency, and frequency) to acoustic stimuli. In general (10 of 14 penetrations), TC patches defined cortical regions of uniform multi-unit response profiles (e.g., BF or binaurality). The patches were not associated with any one group of unvarying response properties (i.e., consistent frequency or binaurality was not always represented in every patch).

An example of anterograde labeled patches in AI resulting from a large BDA injection into the MGV of animal CM-15 is illustrated in Figure 2 (left). In this case, the BDA injection labeled an elongated slab-like region whose orientation paralleled the cellular laminas in the rabbit MGV (Cetas et al., 2001, 2002b). The TC projections labeled by this injection consisted of four

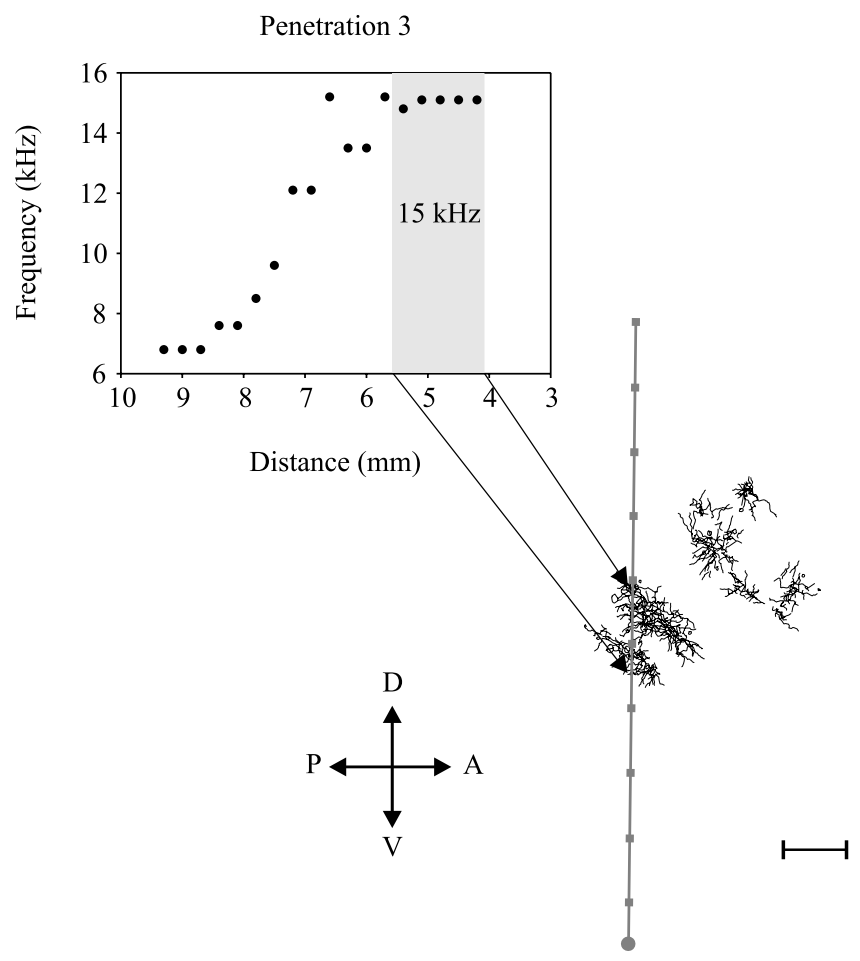

Figure 5. Small TC patches are associated with regions of consistent BF. Reconstruction of electrode penetration through TC axonal fields from experiment CM-27. The graph depicts BFs as a function of electrode depth throughout penetration 3. The shaded area represents the portion of the penetration that passed through the TC patch. Note the consistent $\mathrm{BF}(15 \mathrm{kHz})$ and binaural responses (EE) within the patch and the change in frequency as the electrode exits the patch. $A$, Anterior; $D$, dorsal; $P$, posterior; $V$, ventral. Scale bar, $1.0 \mathrm{~mm}$.

distinct patches shown in Figure 2 (right). A comparison with Figure 1 indicates that the overall orientation of the patches parallels the isofrequency contours of this species (Fig. 2). This relationship was observed in several experiments (see below); it sug- 


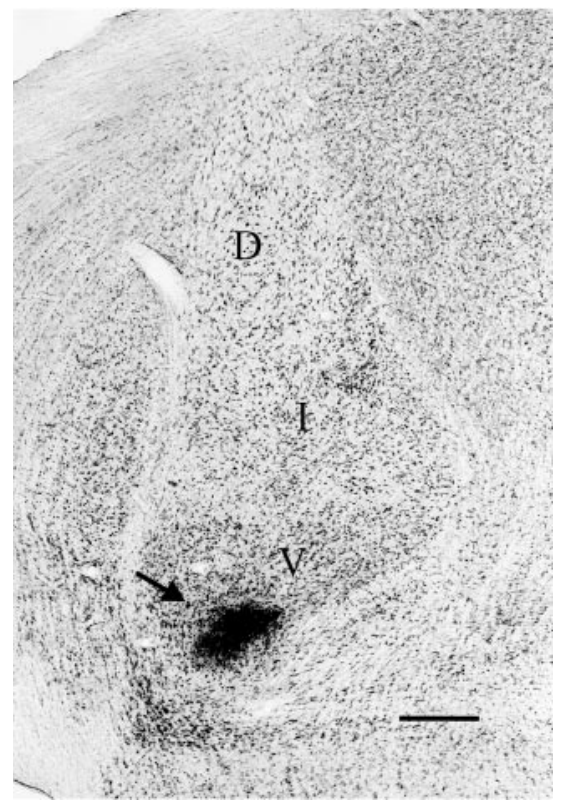

Figure 6. Methylene blue counterstained coronal section through the right MGB in experiment $C M-13$ showing BDA injection (arrow) in the high-frequency region of MGV. D, Dorsal subdivision of the MGB; I, internal division of the MGB. Scale bar, $500 \mu \mathrm{m}$.

gests a point-to-strip organization of TC projections in this species. A reconstruction of the mapping experiment for animal CM-15 is shown in Figure 3. A high-to-low frequency progression was seen during mapping experiments, as revealed by the graphs for penetrations 4 and 5 shown in Figure 3. A dorsal tonotopic field was also seen in this experiment, characterized by a steep low-to-high frequency progression that mirrored the frequency organization of AI (Fig. 3). In experiment CM-15, frequency was consistent throughout mapped portions of each of the TC patches. Binaurality, in the form of EE, was also consistent in each of the penetrations through the TC patches (Fig. 3).

Figure 4 illustrates a coronal section of the MGV from animal $\mathrm{CM}-27$, which received a small BDA injection located at the lateral edge of the anterior MGV. A computer microscope reconstruction of the small TC terminal fields labeled by this injection, along with a reconstruction of one tangential electrode penetration, is shown in Figure 5. Typical for small patches labeled by injections of this size, $\mathrm{BF}(15 \mathrm{kHz})$ and binaurality $(\mathrm{EE})$ remained consistent within the TC patch, whereas threshold and PSTH response type varied (Fig. 5). Note also the posterodorsal to anteroventral orientation of the two small patches shown in the reconstruction of penetration 3 , an orientation that parallels the isofrequency contours of the rabbit AI (Fig. 1).

Figure 6 is a photomicrograph of a larger BDA injection in the high-frequency area of the MGV for experiment CM-13. Similar to CM-15, shown in Figure 2, the BDA injection site has a dorsomedial-ventrolateral orientation similar to that of cellular laminas and frequency slabs that have been described in the MGV (Cetas et al., 2001). A computer microscope reconstruction of TC terminal fields along with reconstructions of two tangential electrode penetrations in auditory cortex from experiment CM-13 are shown in Figure 7. Because of the large BDA injection in the MGV, the TC terminal fields were larger here relative to those

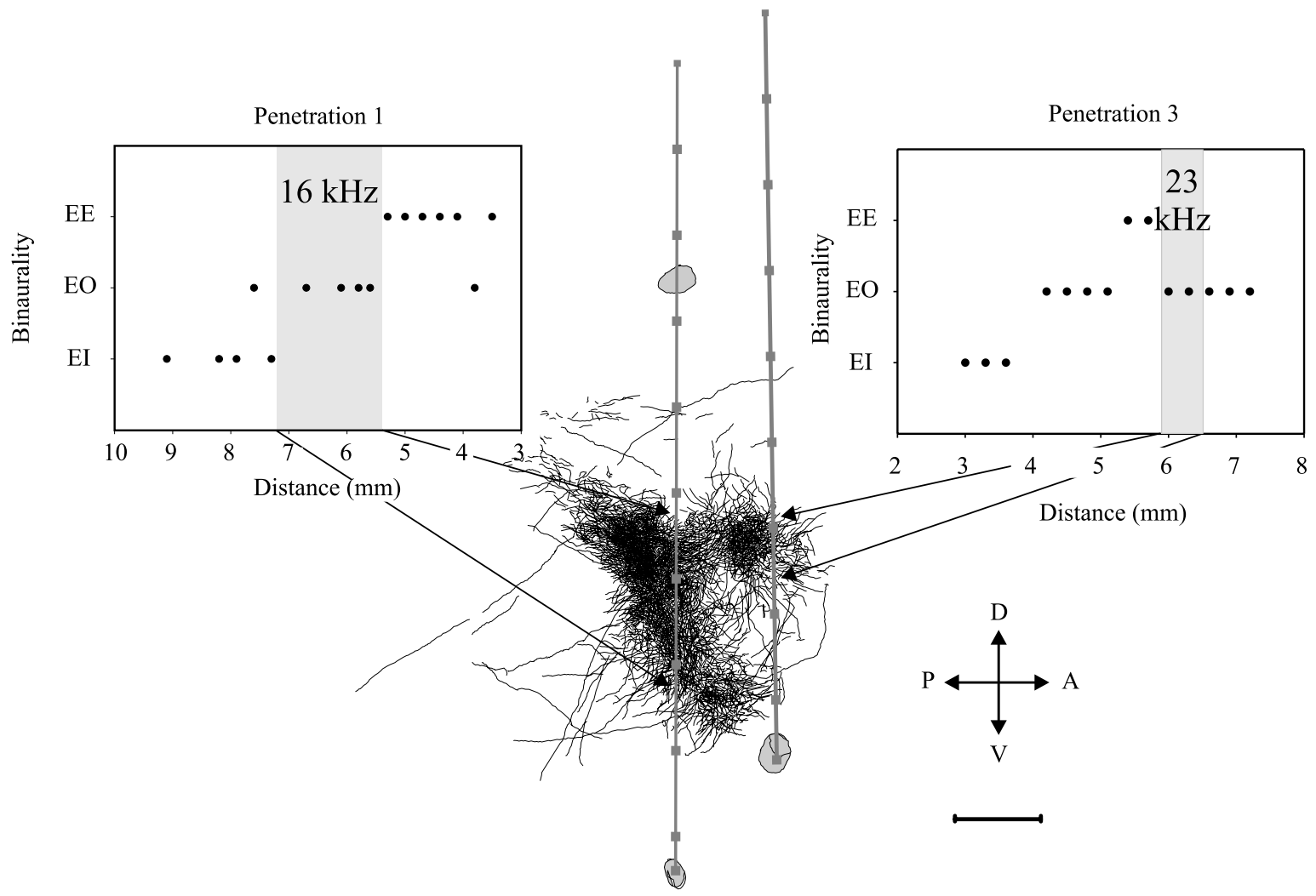

Figure 7. TC patches are associated with areas of consistent binaurality. Reconstruction of two electrode penetrations through elongated TC bands from experiment CM-13. The graphs depict binaurality (EE, E0, El) as a function of depth throughout the penetrations. The graph on the leftrepresents binaurality throughout penetration 1; the graph on the rightrepresents the same for penetration 3 . The shaded arearepresents the portion of penetrations 1 and 3 that passed through TC fields. Note the consistent binaurality within the patches and the change in binaurality at patch borders. BF was constant within each mapped patch. $A$, Anterior; $D$, dorsal; $P$, posterior; $V$, ventral. Scale bar, $1.0 \mathrm{~mm}$. 


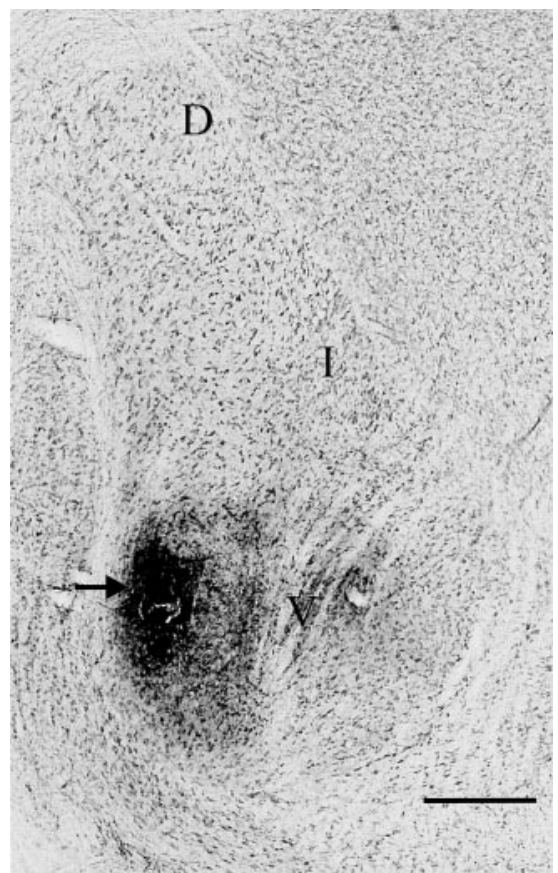

Figure 8. Methylene blue counterstained coronal section through the right MGB showing a large BDA injection (arrow) in the lateral MGV (V).D, Dorsal subdivision of the MGB; I, internal division of the MGB. Scale bar, $500 \mu \mathrm{m}$. from experiment CM-27 (Fig. 5). Similar to those of experiments CM-15 and CM-27 (Figs. 3, 5), the TC terminal fields were oriented in an anteroventral-to-posterodorsal direction. In each penetration, both $\mathrm{BF}$ and binaurality remained constant within the TC axonal field. In penetration 1, a change in binaurality occurred on entering and exiting the TC field. A consistent BF (16 $\mathrm{kHz}$ ) and binaurality (EO) were observed within the patch. A consistent $\mathrm{BF}(23 \mathrm{kHz})$ and binaural response (EO) were also seen in the small patch mapped in penetration 3 (Fig. 7, right). Although frequency remained consistent in both TC fields, the BFs were not the same ( 16 and $23 \mathrm{kHz}$ ). It is interesting to note that $23 \mathrm{kHz}$ is $\sim 0.7$ octaves above $16 \mathrm{kHz}$, a frequency step size that has been described in the rabbit MGB (Cetas et al., 2001).

The BDA injection in the MGV for experiment CM-29 is shown in Figure 8. Unlike the MGV injections in experiments CM-15 (Fig. 3) and CM-13 (Fig. 6), the injection focus extends vertically across the MGV laminas. Such an injection would be expected to cross multiple frequency slabs in this species (Cetas et al., 2002b).

A computer reconstruction of the large terminal TC field labeled by the BDA injection shown in Figure 8 (CM-29) is shown in Figure 9. Note the anteroventral-to-posterodorsal orientation of the TC field, similar to that of CM-15 (Fig. 3) and CM-27 (Fig. 5). Multi-unit responses to acoustic stimulation were more robust within the terminal field than those outside the TC arbors (Fig. 9). The multi-unit response type (onset/sustained) is also consistent throughout the labeled terminal field. A stepwise frequency progression was observed within the TC patch. Binaurality was also consistent throughout the patch, with the majority being classified as EO.

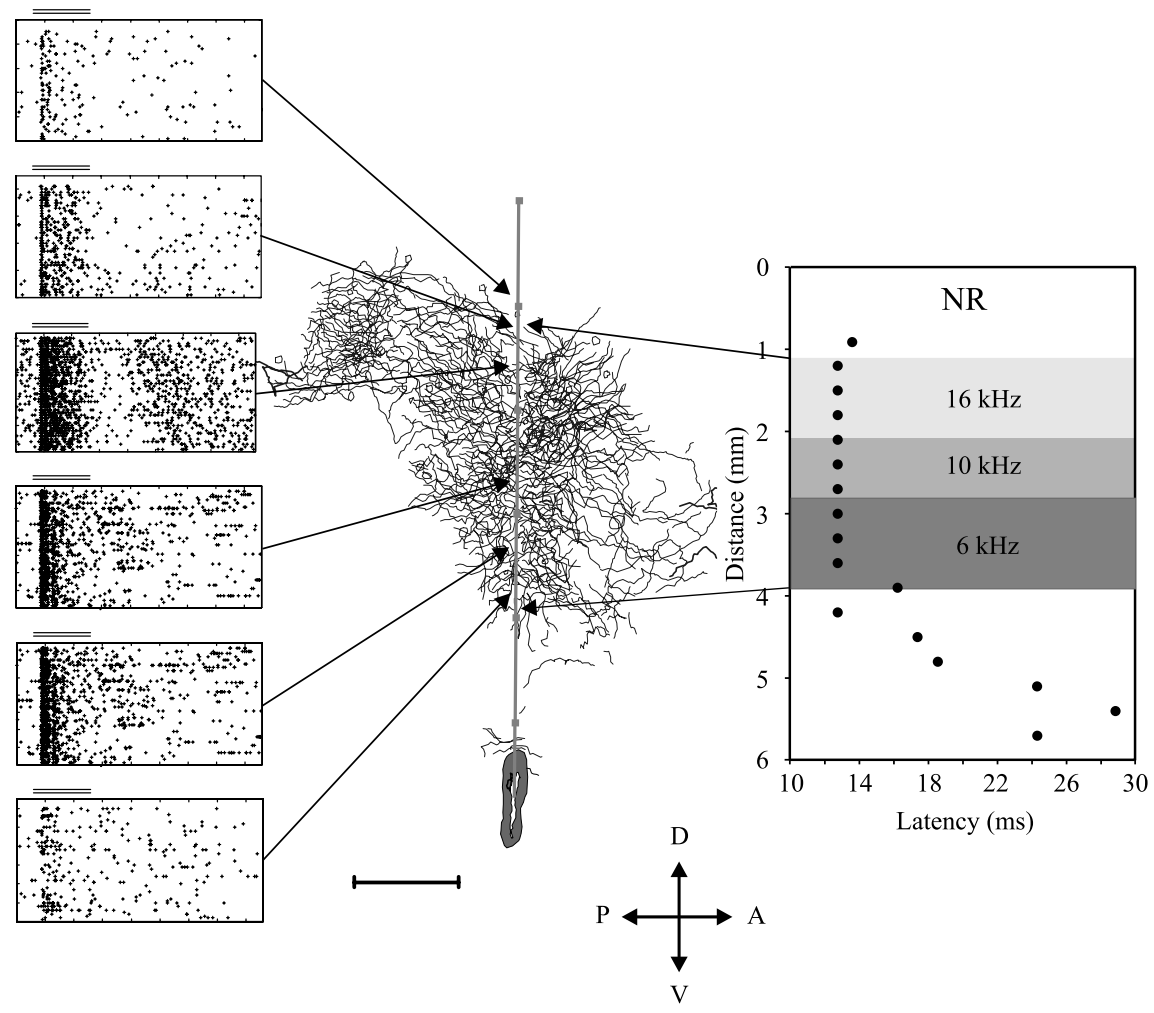

Figure 9. Reconstruction of an electrode penetration through a large TC terminal field from experiment CM-29. Rasters to the left of the penetration indicate multi-unit response patterns at discrete recording points. Note that the type and strength of multi-unit responses change at the patch borders and remain consistent within the patch. The graph on the right depicts response latency throughout the penetration. The shaded area represents the portion of the penetration through the TC patch. Frequency progression was stepwise in this patch; binaurality was consistent (E0). Note the consistent short-latency responses within the patch. Outside the patch, regions of longer latency or no response (NR) predominate. $A$, Anterior; $D$, dorsal; $P$, posterior; $V$, ventral. Scale bar, $1.0 \mathrm{~mm}$.

\section{Discussion}

\section{Auditory fields and TC patches}

Although physiological (Colwell and Merzenich, 1975; Middlebrooks et al., 1980) and anatomical (Middlebrooks and Zook, 1983; Angelucci et al., 1993; McMullen and de Venecia, 1993; de Venecia and McMullen, 1994; Hashikawa et al., 1995; Cetas et al., 1999) evidence for the existence of multiple parallel pathways linking the auditory thalamus and AI has existed for years, there have been few attempts to correlate auditory physiological maps with TC circuits (Middlebrooks and Zook, 1983). In the present study, focal tracer injections in the MGV frequently resulted in a band of TC axons composed of multiple patches (McMullen and de Venecia, 1993). A consistent finding in the present study was that TC patches defined a cortical module with a particular BF. Electrophysiological mapping of several patches making up a band revealed a common BF within the band (experiment CM-15). In most cases, the TC axonal fields were aligned parallel to isofrequency contours in $\mathrm{AI}$, an arrangement predicted by Tunturi (1950) more than 50 years ago. These results are consistent with anterograde and retrograde studies of auditory thalamic pathways that have shown that restricted areas of the MGV 


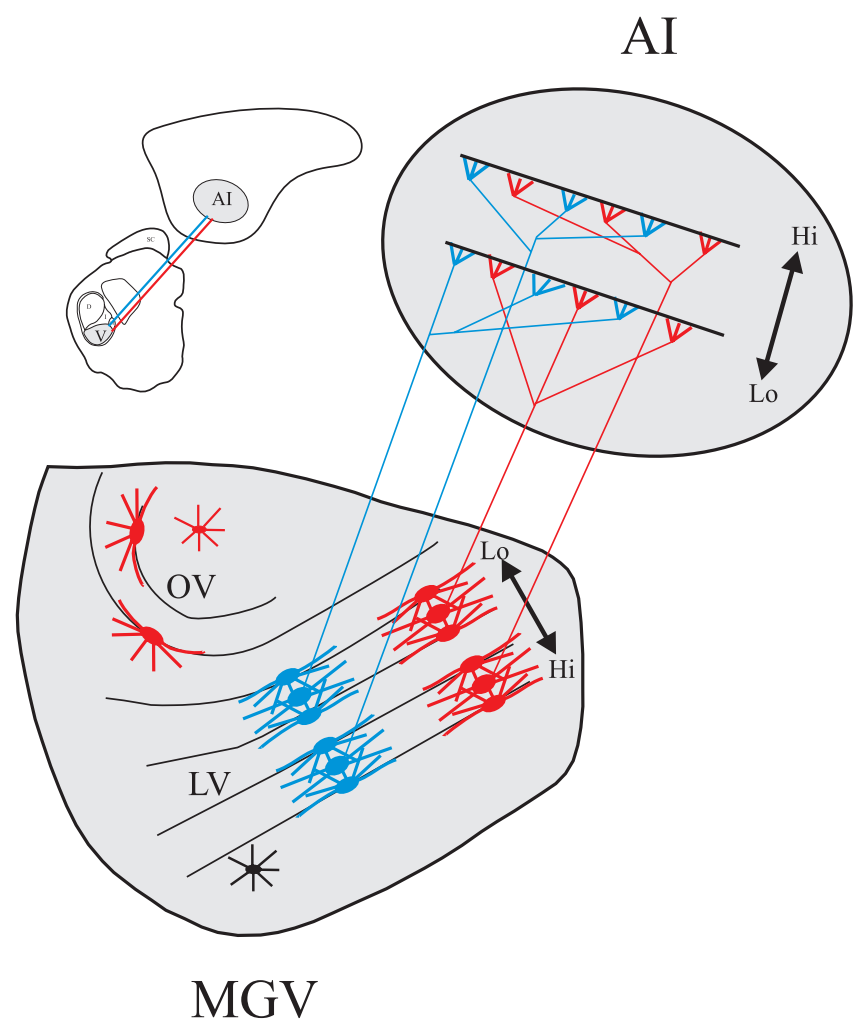

Figure 10. Inset, Diagram of TC projection from the ventral nucleus of the MGB to AI. D, Dorsal division; I, internal division; SC, superior colliculus. Main diagram, Schematic of the MGV showing ovoidea (OV) and laminated (LV) regions (Cetas et al., 2001, 2002a, 2002b). TC axons originating from binaural-specific relay neurons (red, blue) located in frequency slabs terminate in patches along a frequency strip in Al. Adjacent MGB neurons within the same frequency slab but coding for a different binaural class project to separate fields with the same frequency strip. This model explains how continuous and discontinuous maps in Al derive from patchy TC circuits.

diverge to widespread areas along an isofrequency contour (Colwell and Merzenich, 1975; Merzenich et al., 1982; McMullen and de Venecia, 1993; Cetas et al., 1999). We conclude that functionally defined isofrequency contours in AI derive from the patchy divergent projections of auditory thalamic neurons.

Binaural interaction bands form an additional functional map in AI (Imig and Adrian, 1977; Middlebrooks et al., 1980). Neuronal groups projecting to either EE or EI bands are spatially segregated from one another within the MGV (Middlebrooks and Zook, 1983), suggesting the existence of several classes of MGV neurons with unique projections to AI. In the present study, clusters of binaural regions were found within AI composed of EE, EO, or EI response types, which crossed isofrequency contours. The distribution of binaural classes in the rabbit AI is similar to what has been described in AI of several other species, such as the cat (Imig and Adrian, 1977; Middlebrooks et al., 1980; Schreiner, 1991, 1995) and rat (Kelly and Sally, 1988), but has not been reported before in this species. TC patches were also associated with the clustered distribution of binaural classes in AI. In many cases, the patch boundaries defined the borders of binaural transitions (Fig. 7, EI-EO, EO-EE). Our results suggest that TC patches represent the anatomical substrate for frequency and binaural maps in AI. Several other discontinuous maps in the AI overlay the tonotopic and binaural maps and include sharpness of tuning (Schreiner, 1991; Recanzone et al., 1999), intensity tuning (Schreiner et al., 1992; Recanzone et al., 1999), latency (Recanzone et al., 1999), and temporal response properties
(Schreiner, 1991; Schulze et al., 1997). How these maps relate to TC patches requires additional study.

In experiment CM-29, at least three distinct BFs were represented within a large TC field. In contrast, binaurality (as well as latency and response profile) remained consistent. We believe this finding is related to the number of frequency laminas encompassed by the MGV injection (Cetas et al., 2001). The frequency steps within the TC axonal field ( 0.7 octave) were identical to those observed in mapping penetrations across cell laminas in the MGV (Cetas et al., 2001). The constancy of binaural responses within the large patches is consistent with the distribution of binaural classes in the auditory thalamus. In the MGV of the rabbit (Cetas et al., 2001) and cat (Rodrigues-Dagaeff et al., 1989), binaural response classes are mapped independently of frequency.

\section{Auditory cortical maps in the rabbit: comparison with other species}

In our experiments, all TC patches but one were found in areas with a frequency organization that was typical of what has been reported in AI of the rabbit: a dorsoventral high-to-low frequency progression (McMullen and Glaser, 1982). This was expected, because our injections were made in the MGV, the source of the main lemniscal pathway to AI (Winer et al., 1977; Niimi and Matsuoka, 1979; Imig and Morel, 1983; McMullen and de Venecia, 1993). In AI of the rabbit, the tonotopic map and its representative isofrequency contours appear rotated $90^{\circ} \mathrm{com}-$ pared with that in cats and monkeys (Merzenich et al., 1973; Imig et al., 1977; Reale and Imig, 1980; Schreiner, 1991; Recanzone et al., 1999). This rotation is consistent with the relative orientation of cellular laminas in the MGV (Morest, 1965; Imig and Morel, 1984; Cetas et al., 2001). A tonotopic map with isofrequency contours similar to that of rabbits has been reported in an Australian marsupial (Aitkin et al., 1986) and in ferrets (Angelucci et al., 1993). In most mammalian species, the primary auditory field is surrounded by one or more tonotopically organized areas (Tunturi, 1950; Merzenich and Brugge, 1973; Imig et al., 1977; Reale and Imig, 1980; Redies et al., 1989; Hackett et al., 1998). In the rabbit, a secondary field with a tonotopic organization that mirrors AI lies dorsal to AI. Based on its frequency organization and its position relative to AI, this field appears to be the homolog to the anterior auditory field of the cat (Reale and Imig, 1980) and the dorsal caudal field of the guinea pig (Redies et al., 1989; Wallace et al., 2000).

\section{Integrity of tracer-injected MGV}

An important concern when performing combined anterograde labeling and mapping experiments is the possibility that the tracer used to label anatomical structures may damage or destroy cells at the injection site. In addition, uptake, transport, and distribution of the tracer could modify the transmission of information to axonal targets, thus altering the response profile of cortical sites coextensive with the labeled axons. The evidence that BDA injections into the MGV and its transport to AI did not result in alterations in the functional properties of AI include the following: (1) The cortical frequency maps obtained in our studies were very similar to those determined in previous mapping studies of NZW rabbits (McMullen and Glaser, 1982). (2) More importantly, experimental animals served as their own controls; adjacent cortical penetrations in which one electrode passed through a TC patch while the other did not were comparable in frequency progression, latency, sharpness of tuning, and response strength. (3) In some cases, the multi-unit responses within a TC patch 
were more exuberant than those outside the patch. One would expect the opposite effect if the BDA had eliminated excitatory input to cortical neurons. (4) In several cases, the BF of the injection site was represented within the area of a mapped TC patch. If neurons at the MGV injection site had been damaged or destroyed, we would not expect to see the BF of that area represented within a TC patch. In fact, we might expect to see areas of no response or frequency gaps during cortical mapping, but this was not the case. We conclude that the cortical response properties were not significantly affected by the thalamic injections of BDA.

\section{A model of MGB-AI connections}

One of the problems in understanding auditory TC relationships is the difficulty of reconciling continuously distributed functional maps (e.g., frequency) with those exhibiting a discontinuous (e.g., binaural) organization. A model incorporating both of these features of TC circuitry based on anatomical and physiological data (Cetas et al., 1999, 2001, 2002a,b) is shown in Figure 10. In this model, $\mathrm{TC}$ axons originating from binaural-specific relay neurons located in frequency slabs in the MGV terminate in patches along a frequency strip in AI. Adjacent MGB neurons within the same frequency slab but coding for a different binaural class project to separate fields within the same frequency strip. This model explains how continuous and discontinuous maps in AI derive from patchy TC circuits.

\section{References}

Adams JC (1981) Heavy metal intensification of DAB-based HRP reaction product. J Histochem Cytochem 29:775.

Aitkin LM, Irvine DR, Nelson JE, Merzenich MM, Clarey JC (1986) Frequency representation in the auditory midbrain and forebrain of a marsupial, the northern native cat (Dasyurus hallucatus). Brain Behav Evol 29:17-28.

Angelucci A, Clasca F, Sur M (1993) Multiple cortical auditory fields in the ferret defined by their architectonics and thalamocortical connections. Soc Neurosci Abstr 19:1427.

Brugge JF, Imig TJ (1978) Some relationships of binaural response patterns of single neurons to cortical columns and interhemispheric connections of auditory area AI of cat cerebral cortex. In: Evoked electrical activity in the auditory nervous system (Naunton RF, Fernandez C, eds), pp 487503. New York: Academic.

Budinger E, Heil P, Scheich H (2000) Functional organization of auditory cortex in the Mongolian gerbil (Meriones unguiculatus). III. Anatomical subdivision and corticocortical connections. Eur J Neurosci 12:2425-2451.

Cetas JS, de Venecia RK, McMullen NT (1999) Thalamocortical afferents of Lorente de No: medial geniculate axons that project to primary auditory cortex have collateral branches to layer I. Brain Res 830:203-208.

Cetas JS, Price RO, Velenovsky DS, Sinex DG, McMullen NT (2001) Frequency organization and cellular lamination in the medial geniculate body of the rabbit. Hear Res 155:113-123.

Cetas JS, Price RO, Velenovsky DS, Crowe JJ, Sinex DG, McMullen NT (2002a) Cell types and response properties of neurons in the ventral division of the medial geniculate body of the rabbit. J Comp Neurol 445:78-96.

Cetas JS, Price RO, Velenovsky DS, Crowe JJ, Sinex DG, McMullen NT (2002b) Dendritic orientation and laminar architecture in the rabbit auditory thalamus. J Comp Neurol, in press.

Colwell SA, Merzenich MM (1975) Organization of thalamocortical and corticothalamic projections to and from physiologically defined loci within primary auditory cortex in the cat. Anat Rec 181:336.

Cruikshank SJ, Killackey HP, Metherate R (2001) Parvalbumin and calbindin are differentially distributed within primary and secondary subregions of the mouse auditory forebrain. Neuroscience 105:553-569.

de Venecia RK, McMullen NT (1994) Single thalamocortical axons diverge to multiple patches in neonatal auditory cortex. Brain Res Dev Brain Res 81:135-142.

de Venecia RK, Smelser CB, Lossman SD, McMullen NT (1995) Complementary expression of parvalbumin and calbindin D-28k delineates subdivisions of the rabbit medial geniculate body. J Comp Neurol 359:595-612. de Venecia RK, Smelser CB, McMullen NT (1998) Parvalbumin is expressed in a reciprocal circuit linking the medial geniculate body and auditory neocortex in the rabbit. J Comp Neurol 400:349-362.

Fitzpatrick DC, Suga N, Olsen JF (1998) Distribution of response types across entire hemispheres of the mustached bat's auditory cortex. J Comp Neurol 391:353-365.

Hackett TA, Stepniewska I, Kaas JH (1998) Subdivisions of auditory cortex and ipsilateral cortical connections of the parabelt auditory cortex in macaque monkeys. J Comp Neurol 394:475-495.

Hashikawa T, Rausell E, Molinari M, Jones EG (1991) Parvalbumin- and calbindin-containing neurons in the monkey medial geniculate complex: differential distribution and cortical layer specific projections. Brain Res 544:335-341.

Hashikawa T, Molinari M, Rausell E, Jones EG (1995) Patchy and laminar terminations of medial geniculate axons in monkey auditory cortex. J Comp Neurol 362:195-208.

Hendry SH, Jones EG, Emson PC, Lawson DE, Heizmann CW, Streit P (1989) Two classes of cortical GABA neurons defined by differential calcium binding protein immunoreactivities. Exp Brain Res 76:467-472.

Huang CL, Winer JA (2000) Auditory thalamocortical projections in the cat: laminar and areal patterns of input. J Comp Neurol 427:302-331.

Hubel DH, Wiesel TN (1962) Receptive fields, binocular interaction and functional architecture in the cat's visual cortex. J Physiol (Lond) 160:106-154.

Hubel DH, Wiesel TN (1977) Ferrier lecture: functional architecture of macaque monkey visual cortex. Proc R Soc Lond B Biol Sci 8:1-59.

Imig TJ, Adrian HO (1977) Binaural columns in the primary field (A1) of cat auditory cortex. Brain Res 138:241-257.

Imig TJ, Morel A (1983) Organization of the thalamocortical auditory system in the cat. Annu Rev Neurosci 6:95-120.

Imig TJ, Morel A (1984) Topographic and cytoarchitectonic organization of thalamic neurons related to their targets in low-, middle-, and highfrequency representations in cat auditory cortex. J Comp Neurol 227:511-539.

Imig TJ, Ruggero MA, Kitzes LM, Javel E, Brugge JF (1977) Organization of auditory cortex in the owl monkey (Aotus trivirgatus). J Comp Neurol 171:111-128.

Jones EG, Burton H (1976) Areal differences in the laminar distribution of thalamic afferents in cortical fields of the insular, parietal and temporal regions of primates. J Comp Neurol 168:197-247.

Jones EG, Dell'Anna ME, Molinari M, Rausell E, Hashikawa T (1995) Subdivisions of macaque monkey auditory cortex revealed by calciumbinding protein immunoreactivity. J Comp Neurol 362:153-170.

Kelly JB, Sally SL (1988) Organization of auditory cortex in the albino rat: binaural response properties. J Neurophysiol 59:1756-1769.

Kosaki H, Hishikawa T, He J, Jones EG (1997) Tonotopic organization of auditory cortical fields delineated by parvalbumin immunoreactivity in macaque monkeys. J Comp Neurol 386:304-316.

LeDoux JE, Ruggiero DA, Reis DJ (1985) Projections to the subcortical forebrain from anatomically defined regions of the medial geniculate body in the rat. J Comp Neurol 242:182-213.

Marshall WH, Woolsey CN, Bard P (1941) Observations on cortical somatic sensory mechanisms of cat and monkey. J Neurophysiol 4:1-24.

McMullen NT, de Venecia RK (1993) Thalamocortical patches in auditory neocortex. Brain Res 620:317-322.

McMullen NT, Glaser EM (1982) Tonotopic organization of rabbit auditory cortex. Exp Neurol 75:208-220.

McMullen NT, Smelser CB, de Venecia RK (1994) A quantitative analysis of parvalbumin neurons in rabbit auditory neocortex. J Comp Neurol 349:493-511.

Mendelson JR, Schreiner CE, Sutter ML, Grasse KL (1993) Functional topography of cat primary auditory cortex: responses to frequency-modulated sweeps. Exp Brain Res 94:65-87.

Mendelson JR, Schreiner CE, Sutter ML (1997) Functional topography of cat primary auditory cortex: response latencies. J Comp Physiol [A] 181:615-633.

Merzenich MM, Brugge JF (1973) Representation of the cochlear partition of the superior temporal plane of the macaque monkey. Brain Res 50:275-296.

Merzenich MM, Knight PL, Roth GL (1973) Cochleotopic organization of primary auditory cortex in the cat. Brain Res 63:343-346.

Merzenich MM, Colwell SA, Andersen RA (1982) Thalamocortical and cor- 
ticothalamic connections in the auditory system of the cat. In: Cortical sensory organization III (Woolsey CN, ed), pp -57. Clifton, NJ: Humana.

Mesulam MM, Pandya DN (1973) The projections of the medial geniculate complex within the sylvian fissure of the rhesus monkey. Brain Res 60: 315-333. arsid5590278

Middlebrooks JC, Zook JM (1983) Intrinsic organization of the cat's medial geniculate body identified by projections to binaural response-specific bands in the primary auditory cortex. J Neurosci 3:203-224. arsid5590278

Middlebrooks JC, Dykes RW, Merzenich MM (1980) Binaural responsespecific bands in primary auditory cortex (AI) of the cat: topographical organization orthogonal to isofrequency contours. Brain Res 181:31-48.

Molinari M, Dell'Anna ME, Rausell E, Leggio MG, Hashikawa T, Jones EG (1995) Auditory thalamocortical pathways defined in monkeys by calciumbinding protein immunoreactivity. J Comp Neurol 362:171-194.

Morest DK (65) The laminar structure of the medial geniculate body of the cat. J Anat Lond 99:143-160.

Morino-Wannier P, Fujita SC, Jones EG (1992) GABAergic neuronal populations in monkey primary auditory cortex defined by co-localized calcium binding proteins and surface antigens. Exp Brain Res 88:422-432.

Niimi K, Matsuoka H (1979) Thalamocortical organization of the auditory system in the cat studied by retrograde axonal transport of horseradish peroxidase. Adv Anat Embryol Cell Biol 57:1-56.

Reale RA, Imig TJ (1980) Tonotopic organization in auditory cortex of the cat. J Comp Neurol 192:265-291.

Recanzone GH, Schreiner CE, Sutter ML, Beitel RE, Merzenich MM (1999) Functional organization of spectral receptive fields in the primary auditory cortex of the owl monkey. J Comp Neurol 415:460-481.

Redies H, Sieben U, Creutzfeldt OD (1989) Functional subdivisions in the auditory cortex of the guinea pig. J Comp Neurol 282:473-488.

Rodrigues-Dagaeff C, Simm G, De Ribaupierre Y, Villa A, De Ribaupierre F, Rouiller EM (1989) Functional organization of the ventral division of the medial geniculate body of the cat: evidence for a rostral-caudal gradient of response properties and cortical projections. Hear Res 39:103-126.

Romanski LM, LeDoux JE (1993) Organization of rodent auditory cortex: anterograde transport of PHA-L from MGB to temporal neocortex. Cereb Cortex 3:499-514.

Schreiner CE (1991) Functional topographies in the primary auditory cortex of the cat. Acta Otolaryngol Suppl (Stockh) 491:7-16.

Schreiner CE (1992) Functional organization of the auditory cortex: maps and mechanisms. Curr Opin Neurobiol 2:516-521.
Schreiner CE (1995) Order and disorder in auditory cortical maps. Curr Opin Neurobiol 5:489-496.

Schreiner CE, Mendelson JR (1990) Functional topography of cat primary auditory cortex: distribution of integrated excitation. J Neurophysiol 64:1442-1459.

Schreiner CE, Mendelson JR, Sutter ML (1992) Functional topography of cat primary auditory cortex: representation of tone intensity. Exp Brain Res 92:105-122.

Schreiner CE, Read HL, Sutter ML (2000) Modular organization of frequency integration in primary auditory cortex. Annu Rev Neurosci 23:501-529.

Schulze H, Ohl FW, Heil P, Scheich H (1997) Field-specific responses in the auditory cortex of the unanaesthetized Mongolian gerbil to tones and slow frequency modulations. J Comp Physiol [A] 181:573-589.

Sousa-Pinto A (1973) Cortical projections of the medial geniculate body of the cat. Adv Anat Embryol Cell Biol 48:1-42.

Tunturi AR (1950) Physiological determination of the arrangement of the afferent connections to the middle ectosylvian auditory area in the dog. Am J Physiol 162:489-502.

Vater M, Braun K (1994) Parvalbumin, calbindin D-28k, and calretinin immunoreactivity in the ascending auditory pathway of horseshoe bats. J Comp Neurol 341:534-558.

Velenovsky DS, Cetas JS, Price RO, McMullen NT (2000) Thalamocortical patches represent isofrequency regions with consistent binaural response classes: a combined anterograde labeling and mapping study. Abstr Midwinter Res Meet Assoc Res Otolaryngol 23:794.

Velenovsky DS, Cetas IS, Price RO, Sinex DG, McMullen NT (2001) Correspondence between functional subregions and thalamocortical terminal fields in $\mathrm{AI}$ arising from the ventral division of the medial geniculate body. Abstr Midwinter Res Meet Assoc Res Otolaryngol 24:171.

Wallace MN, Kitzes LM, Jones EG (1991) Chemoarchitectonic organization of the cat primary auditory cortex. Exp Brain Res 86:518-526.

Wallace MN, Rutkowski RG, Palmer AR (2000) Identification and localisation of auditory areas in guinea pig cortex. Exp Brain Res 132:445-456.

Winer JA, Diamond IT, Raczkowski D (1977) Subdivisions of the auditory cortex of the cat: the retrograde transport of horseradish peroxidase to the medial geniculate body and posterior thalamic nuclei. J Comp Neurol 176:387-417.

Woolsey CN, Walzl EM (1942) Topical projection of nerve fibers from local regions of the cochlea to cerebral cortex of the cat. Bull Johns Hopkins Hosp 71:315-344. 